\title{
Medical journals and industry ties
}

\author{
Zero tolerance on education articles with financial links to industry
}

\author{
Mabel Chew practice editor, Catherine Brizzell head of education, Kamran Abbasi international \\ editor, Fiona Godlee editor in chief
}

The BMJ, London WC1H 9JR, UK

The BMJ was one of the first medical journals to seek declarations of competing interests from authors. Our focus is on financial competing interests as we believe these to be the most identifiable. We do, however, understand that competing interests come in many forms and we also routinely ask authors to declare relevant non-financial competing interests. The governing principle has been that transparency is a panacea. ${ }^{1}$ We placed faith in this principle, but mounting experience and evidence tell us that we were only half right. ${ }^{2}$ Transparency remains essential, but it isn't sufficient to eliminate bias or perception of bias.

We believe this risk of bias is particularly important for clinical educational articles that are designed to guide patient care, when authors' biases may be less visible to general medical readers. For some years we have sought to minimise as well as declare competing interests for these articles. Recently we introduced more active management of competing interests, requiring authors to complete a more detailed declaration and excluding authors with close ties. Now we have decided to go a step further, as heralded three years ago. ${ }^{3}$ From next year our clinical education articles will be authored by experts without financial ties to industry (box). By industry we mean companies producing drugs, devices, or tests; medical education companies; or other companies with an interest in the topic of the article. We are phasing in this policy to start with editorials, clinical reviews, and most practice series. We hope that by the end of 2016, this will have extended to the rest of our education section: our specialist state of the art reviews and diagnostics and therapeutics series.

\section{Shift in culture}

Why are we doing this? The first reason is that making clinical decisions based on information biased by commercial interests can cause harm, as happened with cardiotoxicity from rosiglitazone and rofecoxib ${ }^{56}$ and continues to happen with hydroxyethyl starch. ${ }^{7}$ We also believe that the educational content we publish will have more impact if readers can trust it. We know that readers consider research papers written by authors with declared financial links to industry to be less important, relevant, rigorous, and believable ${ }^{89}$; they are also less willing to prescribe drugs evaluated in such papers. ${ }^{9}$ Finally, we want to encourage a shift in the culture of medicine. We think that we can help to do this by promoting authors without financial ties to industry and offering them appropriate prominence and visibility.
Financial competing interests are endemic to the culture of medicine and are rarely driven by malign motives or actions. The mechanisms of influence are diverse. An author of a review article might be an advisory board member for companies selling drugs for that condition, a commentator might have received honorariums from industry for lectures on the topic, or an editorialist on a disease might be a patent holder for one of its diagnostic tests. Psychological research suggests that biases may operate subconsciously. ${ }^{10}$ Our decisions not to proceed with an article or an author are not made lightly. Nor are they intended to pass judgment on an author's integrity. However, we cannot ignore the mounting evidence of systematic attempts by commercial interests to corrupt the literature and influence clinical decisions. Internal company documents revealed during litigation expose practices aimed at influencing clinicians such as funding medical meetings, dinners, studies, and articles. ${ }^{10}$ Many clinical practice guidelines are little more than industry marketing tools because of the financial competing interests of their authors and sponsors. ${ }^{11}$

\section{Making it work}

Will our new policy mean we lose the expertise of those at the cutting edge of research? Are there enough experts free of industry ties to satisfy the needs of a weekly general medical journal? In some fields-for example, obesity medicine, genetics, and rheumatology_we may find it difficult to recruit authors free of relevant financial links with industry. It might even prove impossible. But we believe the ethical arguments are persuasive and that this approach will cause less harm. We will study progress and report back. We are tracking how long and how many approaches it takes to find authors who are free of financial links. We are willing to miss out on articles on a few topics in exchange for publishing more articles by authors with no relevant financial ties to industry. And things should become easier over time: if current trends continue then ties between academics and industry are on the wane. ${ }^{12} \mathrm{We}$ will let you know about the topics we struggle with, which in itself will be educational. We realise that non-financial influences matter. ${ }^{13}$ However, our aim is not to eradicate all competing interests - that would be impossible. Nor do we want to be anti-business just for the sake of it. Rather we wish to focus on ties that are known to, and indeed are largely designed to, influence clinical decisions in favour of industry. 


\section{Competing interest definitions and process for The BMSs editorials and education articles (including clinical reviews, practice articles, and state of the art reviews)}

"A conflict of interest arises when a person has a personal or organisational interest that may influence or appear to influence the work they are doing. Usually this is a financial interest, but it may also be non-financial."

- We ask authors to declare interests in the 36 months before the declaration and those known to be going to occur during the next 12 months

- Authors are asked to complete a form, available at www.bmj.com/sites/default/files/attachments/resources/2011/07/current-bmjeducation-coi-formfinal-1.doc. For unsolicited articles, we also ask who prompted submission and whether professional writers contributed

- Each author's declaration is carefully assessed by the handling editor, and may be discussed at a regular editors' meeting, to ensure our decisions are consistently and fairly applied by the editorial team.

- We have started publishing authors' competing interests forms alongside the articles, and advise authors of this when they send their forms. We plan to do so for all editorials and education articles

- From 2015, we will roll out a policy of editorials and clinical education articles authored by experts without financial ties to industry (companies producing pharmaceuticals, devices, or tests; medical education companies; or other companies with an interest in the article topic)

We can also learn from the experience of other journals. In 2002, the New England Journal of Medicine abandoned a strict policy on authors with industry ties. "Our ability to provide comprehensive, up-to-date information, especially on recent advances in therapeutics, has been constrained," conceded its editors. ${ }^{14}$ However, an editor in chief of NEJM when that policy operated in the 1990s explains how it can be made to work: "Sometimes it required going down the list until we found someone who didn't have a conflict, but we never had to compromise and accept someone without sufficient expertise to do a good job." ${ }^{15}$ For over two decades the journal American Family Physician, which primarily publishes clinical reviews, has not considered articles by authors who have financial ties with industry. ${ }^{16}$

Please let us have your views on this change in The BMJ's editorial policy. Our aims are to preserve and enhance readers' trust in the journal's content and to help to shape a new relationship between journals and industry, rather than perpetuate the perception of medical journals as the marketing arm of commercial interests.

Competing interests: We have read and understood BMJ policy on declaration of interests and have no relevant interests to declare.
Smith R. Beyond conflict of interest. BMJ 1998;317:291.

Wilson M. Is transparency really a panacea? J R Soc Med 2014;107:216-7. Godlee F. Turning the tide on conflicts of interest. BMJ 2011:343:d5147.

BMJ. BMJ policy on conflict of interest. www.bmj.com/sites/default/files/attachments/ resources/2011/07/bmjpolicyondeclarationofinterestsmarch2014.pdf.

5 Krumholz H, Ross JS, Presler AH, Egilman DS. What have we learnt from Vioxx? BMJ 2007;334:120

6 Moynihan R. Rosiglitazone, marketing, and medical science. BMJ 2010;340:c1848.

7 Hartog CS, Natanson C, Sun J, Klein HG, Reinhart K. Concerns over use of hydroxyethyl starch solutions. BMJ 2014;349:95981.

8 Schroter S, Morris J, Chaudhry S, Smith, R, Barratt H. Does the type of competing interest affect readers' perceptions of the credibility of research? Randomised trial. BMJ 2004;328:742.

9 Kesselheim AS, Robertson CT, Myers JA, Rose SL, Gillet V, Ross KM, et al. A randomized study of how physicians interpret research funding disclosures. N Engl J Med 2012;367:1119-27.

10 Lo B, Field MJ, Institute of Medicine (US) Committee on Conflict of Interest in Medical Research, Education, and Practice, eds. Conflict of interest in medical research, education, and practice. National Academies Press, 2009.

11 Lenzer J, Hoffman JR, Furberg CD, loannidis JPA. Ensuring the integrity of clinical practice guidelines: a tool for protecting patients. BMJ 2013;347:f5535.

12 Zinner DE, Bolcic-Jankovic D, Clarridge B, Blumenthal D, Campbell EG. Participation of academic scientists in relationships with industry. Health Affairs 2009;28:1814-25.

13 Smith R, Feachem R, Feachem NS, KoehImoos TP, Kinlaw $H$. The fallacy of impartiality: competing interest bias in academic publications. J R Soc Med 2009;102:44

14 Drazen JM, Curfman GD. Financial associations of authors. N Engl J Med 2002;346:1901-2.

15 Kassirer JP. What the New England Journal of Medicine did. BMJ 2011;343:d5665.

16 American Academy of Family Physicians. Conflict of interest form. www.aafp.org/dam AAFP/documents/journals/afp/COlform-2012.pdf.

Cite this as: BMJ 2014;349:97197

(๑) BMJ Publishing Group Ltd 2014 